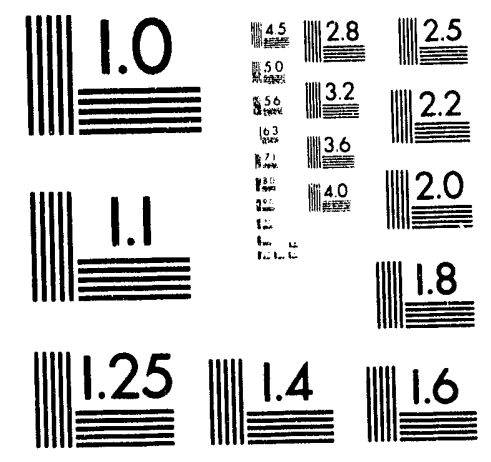



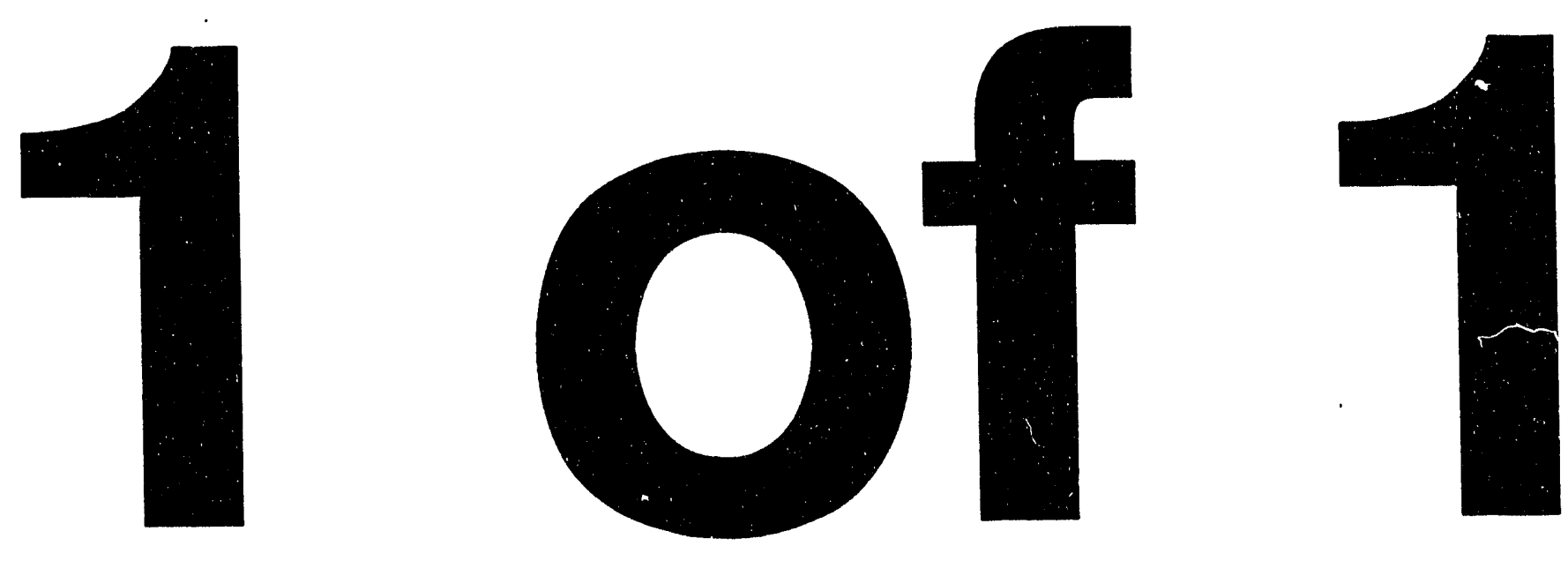


\title{
X-Ray Beam Lines and Beam Line Components for the SLAC Linac Coherent Light Source (LCLS)*
}

\author{
R. Tatchyn and P. Pianetta \\ Stanford Linear Accelerator Center, Stanford, CA 94305, USA
}

\section{Abstract}

The LCLS is a novel high-brightness $x$-ray source designed to operate in the $300-400 \mathrm{eV}$ range. In contrast to conventional synchrotron radiation sources, its output pulses will be characterized by unprecedented levels of brevity and peak power. In this paper we present recently-developed beam line layouts and design features intended to optimize the delivery of the LCLS photons to various experimental stations.

\section{INTRODUCTION}

The LCLS, a Free-Electron Laser (FEL) operating in the Self-Amplified Spontaneous Emission (SASE) regime [1], is an intense novel $x$-ray source whose high-brightness, subpicosecond pulses are expected to provide new research opportunities in a number of diverse areas of $x$-ray science and technology [2]. A brief list of selected output parameters is tabulated below.

Table 1

Nominal L.CLS Output Parameters

\begin{tabular}{|lr|}
\hline 1st Harmonic Wavelength $(\lambda)$ & $40 \AA-30 \AA$ \\
\hline Peak Power $\left(\mathrm{P}_{\text {peak }}\right)$ & $10 \mathrm{GW}$ \\
\hline Beam Diam. $\left(\mathrm{D}_{\mathrm{w}}\right)$ at Optics Locations & $1 \mathrm{~mm}-2 \mathrm{~mm}$ \\
\hline Full Pulse Duration $\left(\sqrt{2 \pi} \sigma_{\tau}\right)$ & $0.5 \mathrm{ps}$ \\
\hline Full Pulse Length & $150 \mu$ \\
\hline Pulse Repetition Rate & $120 \mathrm{~Hz}$ \\
\hline Energy per Pulse & $\approx 5 \mathrm{~mJ}$ \\
\hline Peak Power Density (Norm. Incidence) & $\sim 10^{16} \mathrm{~W} / \mathrm{m}^{2}$ \\
\hline Source Emittance (Diffraction Limited) & $<20 \AA-\mathrm{rad}$ \\
\hline
\end{tabular}

Evidently, a number of these parameters represent extensions of conventional $x$-ray source characteristics (e.g., peak power and pulse length in 3rd Generation synchrotron storage rings [3]) by several orders of magnitude. The purpose of this paper is to describe design aspects of selected optical components and beam line systems that have been recently studied for delivering the LCLS output radiation to experimental users.

\section{PEAK POWER DAMAGE LIMITATIONS}

From Table 1 it is evident that a photon pulse at normal incidence can deposit of the order of $1 \mathrm{eV}$ per atom for

\footnotetext{
- Supported by DOE Offices of Basic Energy Sciences and High Energy and Nuclear Physics and Department of Energy Contract DEAC03-76SF0015.
}

absorptivities [4] and penetration depths typical of solid state materials in the soft $x$-ray range. Since this level of energy loading can be shown to lead to the enhanced probability of lattice damage [5], an important stratagem for reducing it involves decreasing the angle of incidence, $\theta_{\mathfrak{l}}$, on the optical surface in question. As shown in Fig. 1, this leads to the notion of employing multiple reflections at grazing incidence to deflect the LCLS beam into a desired angle $\theta_{\mathrm{T}}$. For

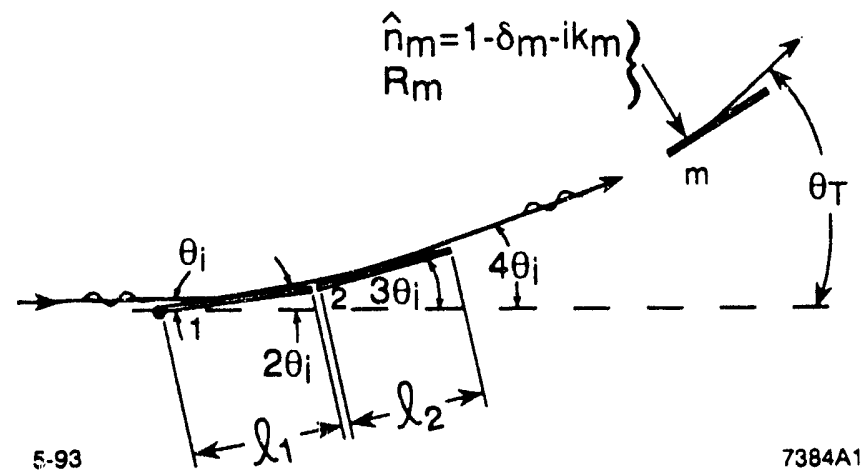

Figure 1. Parameters for multiple grazing-incidence reflection.

reflectors all with equal surface reflectivities $R$, indices of refraction $n=1-\delta+i k$, atomic densities $\#\left[\mathrm{~cm}^{-3}\right]$, and approximate (vertical) penetration depths $\delta_{p}\left(\delta_{p}=\lambda / 4 \pi \sqrt{k}\right)$, a useful figure of merit, $\eta_{A}$ [eV/atom], can be defined [5] from which practical design parameters can be established:

$$
\eta_{A}=\frac{P_{\text {peak }} \sqrt{2 \pi} \sigma_{\tau}}{q}\left[\frac{\theta_{i}}{D_{w}^{2}}\right]\left[\frac{1-R}{\delta_{p}^{\#}}\right] \ll 1
$$

Selecting $\eta_{A} \leq 0.01$, a criterion established by earlier experimental work at SSRL [6], acceptable grazing-incidence angles for $\mathrm{Au}$ (or $\mathrm{Pt}$ ) reflecting surfaces of $\leq 34 \mathrm{mr}$ were arrived at. For future FEL sources with significantly greater levels of peak output power (e.g., tapered-wiggler FELs), the correspondingly smaller angles of incidence predicted by eq. (1) could imply impractically long optical surfaces and alternative methods of beam deflection and processing may need to be considered [7]. The second peak power damage limitation of importance to the present study is associated with the intense electrostatic stresses that will be generated by photoemission from the LCLS optical surfaces. Preliminary assessments indicate that this will necessitate the use of metal substrates for both mirrors and gratings, (to minimize chargeneutralization time intervals) and the elimination of sharpfeatured grooves or profiles on "̈ifraction grating surfaces (to 
reduce the generation of peak field stresses).

\section{BEAM LINE LAYOUT}

As reported elsewhere, the presently proposed location for the LCLS undulator, electron beam dump, and beam lines is at the end of the Final Focus Test Beam (FFTB) enclosure at SLAC [1]. A 1st phase layout of the beam lines and experimental station locations is shown in Fig. 2. The $4^{\circ}$

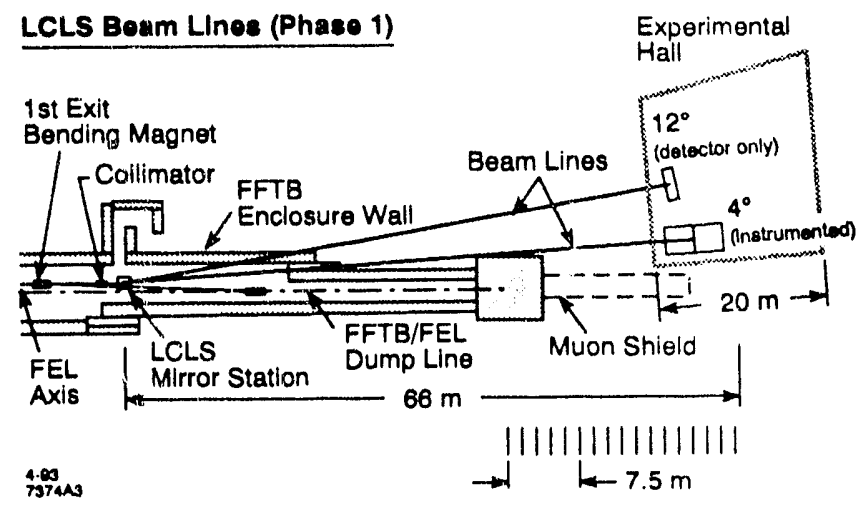

Figure 2. LCLS initial-phase beam lines.

line is planned as a flexible, fully-instrumented research and development facility capable of supporting a variety of initial or demonstration experiments. These will emphasize both basic science and technological applications [2], including the further development of optical instrumentation. The basic purpose of the $12^{\circ}$ line will be to investigate beam-switching techniques and stratr gies that may be utilized in the future to accomodate larger numbers of user end stations. This line will be minimally instrumented, serving primarily to characterize the position and stability of the switched photons.

A more detailed layout indicating essential beamtransport, vacuum, mechanical stabilization, and optical components is shown in Fig. 3.

\section{MECHANICAL AND OPTICAL DESIGN FEATURES}

In view of the long distances involved, small mechanical perturbations of the mirrors will introduce appreciable deflections at the experimental locations. Active suppression of such deviations can be accomplished by detection followed by feedback and coriection of the mirror chamber coordinates. Stabilization with respect to both low and high frequency motion (into the $\mathrm{kHz}$ regime) will be provided.

A major problem experienced by mirror surfaces in synchrotron rediation (SR) installations with vacuums in the $<10^{-9}$ Torr range is the gradual accumulation of carbon [8]. This problem will be compounded in the LCLS due to the fact that its operating energy lies near the carbon K-edge, and even marginal increases in absorptivity (as represented by the 1-R factor ineq. (1)), could lead to serious damage. In consequence, all of the LCLS sections containing optical

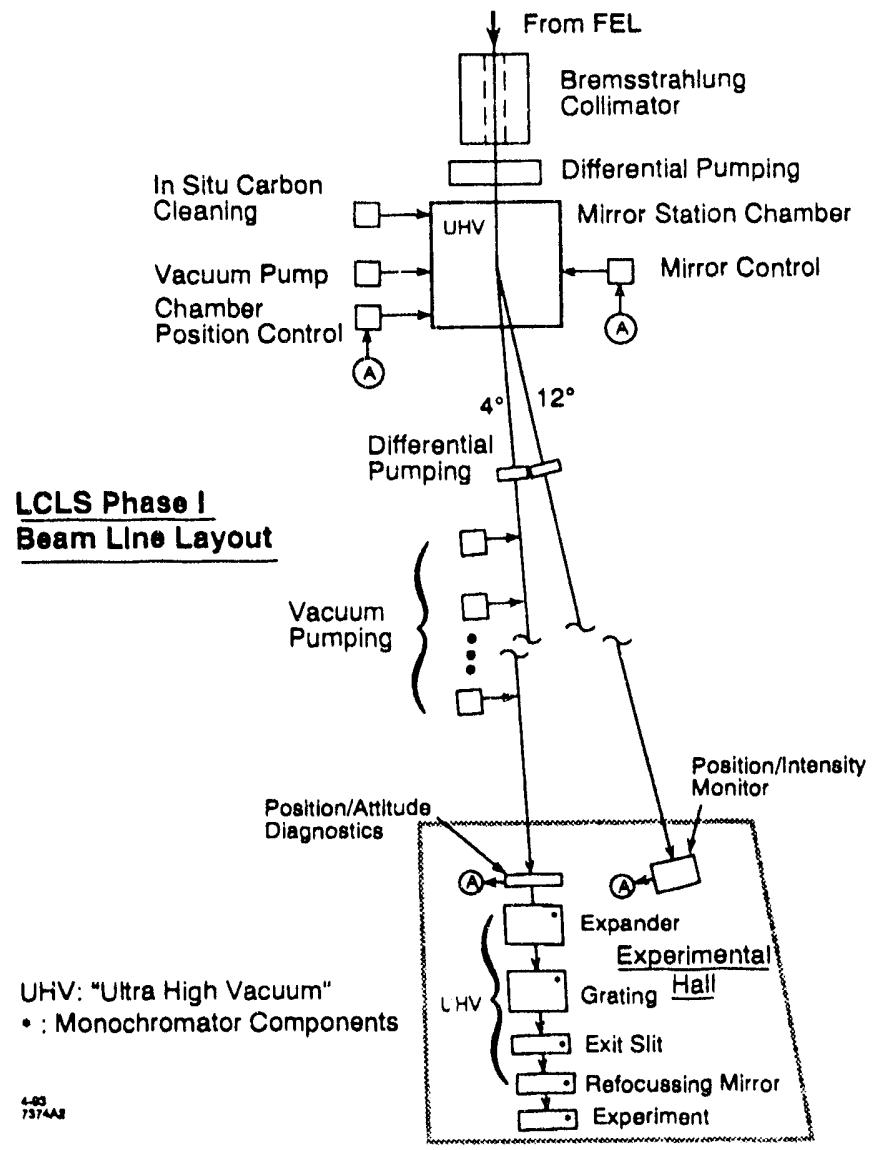

Figure 3. Beam line component layout in block form.

surfaces will need to be maintained as Ultra High Vaculum (UHV) environments. Highly effective differential pumping sections will need to be used to help isolate UHV sections from higher-pressure transport ducts, and provisions for: periodic in situ cleaning of mirror (Fig. 4) and grating surfaces will need to be provided. Due to existing lack of

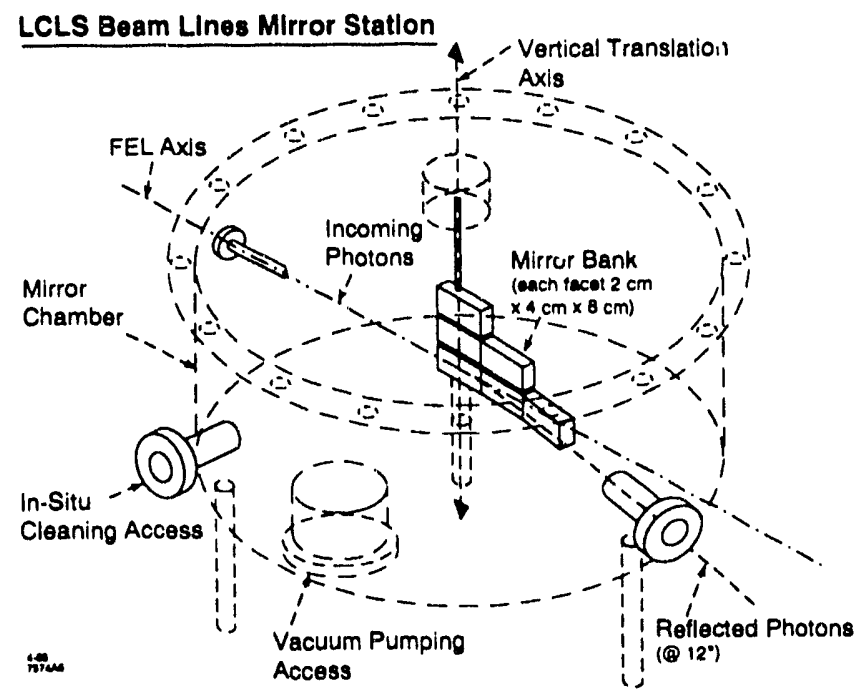

Figure 4. LCLS mirror station tank. 
experience with the effects "bremstrahlung on the carbon contamination of surfaces, th.e installation of a special collimating aperture upstream of the mirror chamber has been proposed.

The mirror station, shown in Fig. 4, features multiple reflectors, vertically arranged to provide switching among different beam lines with a single vertical motion. The mirror facets have been made $4 \mathrm{~cm}$ high to allow several successive vertical translations to unused portions of their surfaces in the event that the rate of surface damage is found to be severe. As may be deduced from Table 1, the time-averaged power impinging on each of the mirror surfaces is less than 1 Watt, and the expected absorbed power should idealiy remain at 1-2 orders of magnitude below this. As a consequence, apart from providing adequate thermal conduction paths to the outside, no specialized heat-removal technology will need to be implemented Regarding the mirrors, presently avaiable polishing techniques can attain surface smoothness down to the $1-2 \AA$ level, which is significantly smaller than the LCLS operating wavelength. Apart from special surface contamination or damage effects, the mirror reflectivity should consequently be insensitive to surface-roughness scattering and should display its approximately ideal value

The monochromator, in view of the unique spectral properties of the LCLS, allows for a number of novel approaches to its design. The nost important simplifying advantage of the LCLS is that the emittance of its source point is, by definition, diffraction linited. This eliminates the need for initial optical processing of the radiation upstream of the monochromator entrance: the source point itself serves as the best possible entrance aperture. This type of design, in principle, allows the use of a single grating with an exit slit followed by a refocussing mirror, a configuration of minimal complexity. Due to the extremely small divergence angle of the LCLS beam, however, provisions have been allowed for expanding the beam prior to diffraction, in order to maximize the attainable resolution. A further refinement necessitated by the peak intensity of the radiation is the mandatory avoidance of diffraction gratings with sharp-featured groove profiles. Furthermore, diffraction with the groo.es placed perpendicularly to the axis of the incoming light constrains the saliency of the (smoothed) grooves even further, since the incidence angle over the entire grating surface should be kept small enough to prevent all but negligible levels of absorption. These constraints have led to the consideration of a sinusoidally-profiled diffraction grating operating in a conical dispersion geometry [9] as perhaps the best candidate solution.

Apart from conventional design issues, further development work on the monochromator is anticipated due to fundamental performance limits stemming from the brevity of the LCLS pulses. In the limit where the photon pulse length begins approaching the coherence length of the monochromatized light, the resolving power of the monochromator becomes attenuated by the effective acceptance of its dipersion aperture $[5,7$, This problem can be mitigated by attempting to lengthen the photon pulses prior to diffraction, either by lengthening the electron bunch in the linac, or by optical means.

\section{ACKNOWLEDGEMENTS}

Valuable technical support and contributions to this project by David Lunt (Photon Sciences Corp.) and Chris Sheppard and Kent Pflibson (Kaman Aerospace Corporation) are gratefully acknowledged. Thanks are offered to all the members of the LCLS research group for their continuing support and interest in this work.

\section{REFERENCES}

[1] H. Winick, K. Bane, R. Boyce, K. Halbach, K.-J. Kim, G. Loew, P. Morton, H.-D. Nuhn, J. Paterson, C. Pellegrini, P. Pianetta, D. Prosnitz, J. Rosenzweig, J. Seeman, T. Raubenheimer, T. Scharlemann, R. Tatchyn, G. Travish, V. Vylet, M. Xie, "A $2.4 \mathrm{~nm}$ Linac Coherent Light Source (LCLS) Using the SLAC Linac," this conference F7, 1993.

[2] W. Spicer, J. Arthur, H. Winick, eds., Proceedings of the Workshop on Scientific Applications of Short Wavelength Coherent Light Sources, "Stanford, CA, October 21, 1992, SLAC-Report-414, February 1993.

[3] M. Cornacchia and H. Winick, eds., Proceedings of the Workshop on Fourth Generation Light Sources, SSRL, Feb. 24-27, 1992, SSRL Pub. 92/02, p. 86.

[4] B. L. Henke, P. Lee, T. J. Tanaka, R. L. Shimabukuro, and B. K. Fujikawa, "The Atomic Scattering Factor, $1+$ if 2 , for 94 Elements and for the 100 to $2000 \mathrm{eV}$ Photon Energy Region," AIP Conference prooceedings No. 75, 340(1982).

[5] R. Tatchyn, "LCLS Optics: Selected Technological Issues and Scientific Opprtunities," ibid., p. 93.

[6] R. Tatchyn, P. Csonka, H. Kilic, H. Watanabe, A. Fuller, M. Beck, A. Toor, J. Underwood, and R. Catura, "Focusing of undulator light at SPEAR with a lacquer-coated mirror to power densities of $10^{9}$ Watts $/ \mathrm{cm}^{2}$," SPIE Proceedings No. 733, 368(1987).

[7] R. Tatchyn, "Photon Pulse Filtering and Modulation Based on the Extreme Temporal Compression and Correlated Energy Snricad of the Electron Bunches in the SLAC Linac Coherent Light Source (LCLS)," this conference - Mb28, 1993.

[8] T. Koide, M. Yanagihara, Y. Aiura, S. Sato, T. Shidara, A. Fujimori, H. Fukutani, M. Niwano, H. Kato, "Resuscitation of carbon-contaminated mirrors and gratings by oxygen-discharge cleaning. 1: Efficiency recovery in the 4-40-eV range," Applied Optics 26(18), 3883(1987).

[9] M. C. Hettrick and S. Bowyer, "Variable line-space gratings: new designs for use in grazing incidence spectrometers," Applied Optics 22, 3921(1983). 

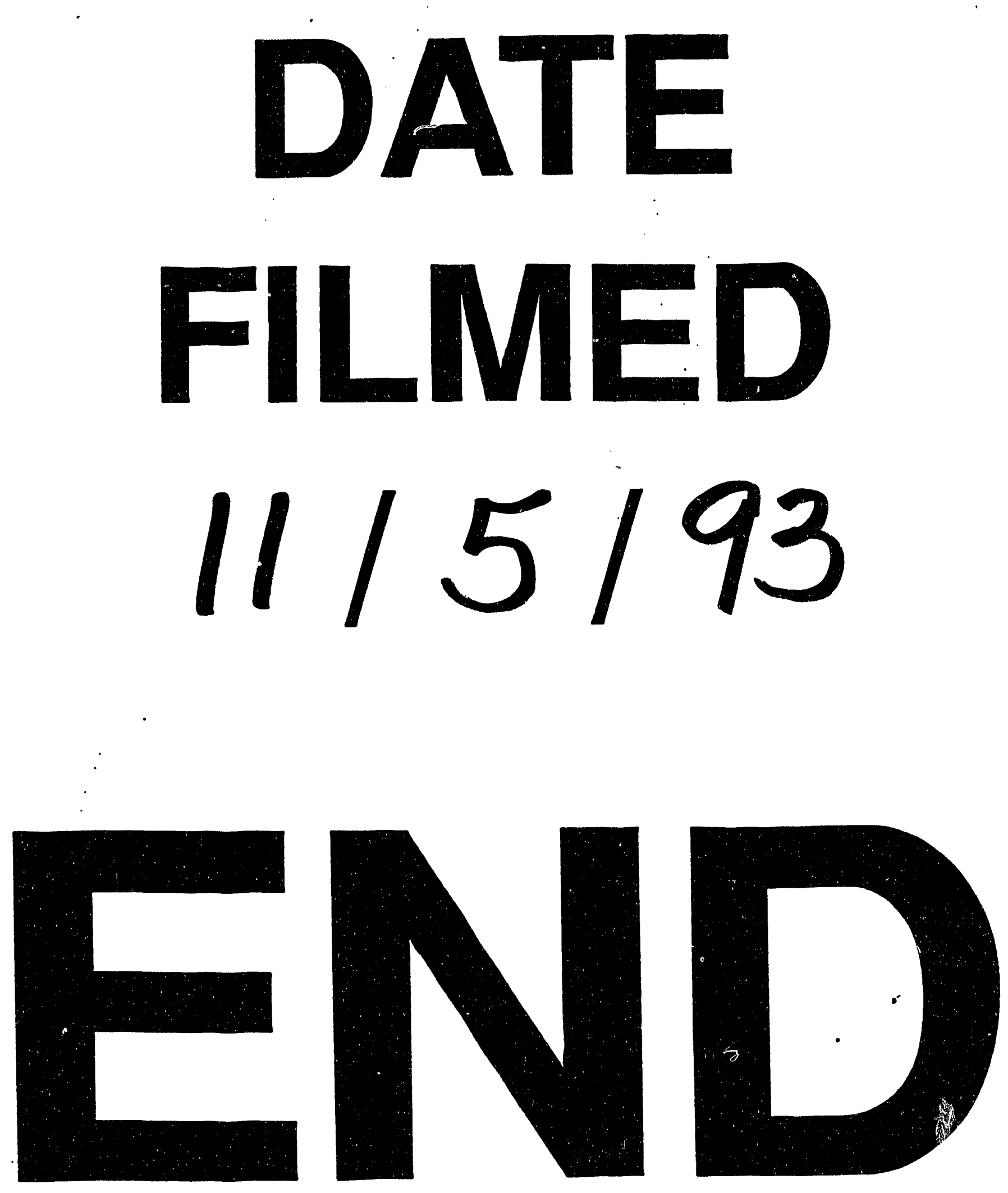
